\title{
Research of Boiler Fault Diagnosis Based on Fuzzy Neural Network
}

\author{
Yifeng $\mathrm{Wu}^{1, \mathrm{a}}$, Xiaoqi Lin $^{2}$ \\ ${ }^{1}$ College of Mechanical Engineering and Automation,Fuzhou University, Fuzhou, 350108 \\ ${ }^{2}$ College of Mechanical Engineering and Automation,Fuzhou University, Fuzhou, 350108 \\ ayifengwu@126.com
}

Keywords: boiler; fuzzy neural network; fault diagnosis

\begin{abstract}
Boiler system has the characteristics of high complexity, strong simultaneity, multiple measuring points, multiple faults, and the traditional fault diagnosis method can not meet the requirements. A fault diagnosis model based on fuzzy neural network is built by the combination of fuzzy logic technology and the improved BP neural network algorithm. The model is used to fault diagnosis and it can better solve the ambiguity, simultaneity and correlation of the boiler fault.Through analyzing the fault example of high temperature superheater damage, and the diagnosis results of the application of the model are verified that they are consistent with the actual operation conditions.
\end{abstract}

\section{Introduction}

Boiler is widely used in the industrial production, but because of its harsh operating environment, the accident rate is higher, resulting in greater harm boiler accident, and the economic losses caused are more serious. Therefore, to ensure safe and stable operation of the boiler equipment, not only can improve the utilization rate of energy and equipment, reduce the repair cost effectively, also can reduce the casualties accidents. The structure of modern boiler system is complex, and operation parameters and fault alarm parameters are more. The operating personnel can't conduct a comprehensive understanding and grasp the alarm information in a short time, and it is difficult to determine the root cause quickly, thus affecting the processing speed of the accident, and extremely easy to cause the production of harm. The cause of boiler fault is usually the interaction of multiple factors, the same fault has different symptoms and there are multiple failures occur simultaneously, so fault diagnosis is difficult. Therefore it is necessary to establish a fault diagnosis system of boiler to provide effective guidance for operating personnel. This paper introduces the design of boiler system fault diagnosis based on fuzzy neural network. The fuzzy neural network algorithm is used to analyze the monitoring data, and the fuzzy mathematics method is used for the high logical concept quantification of the data before input the neural network, then through the neural network reasoning, and obtains the result of fault diagnosis finally.

\section{Fuzzy Pretreatment}

The method to comprehensive analysis and processing for the real-time operation parameters of the boiler field, and extraction of fault symptoms, then judge fault according to the experience from the operating personnel and a specific standard value is too absolute. For example, boiler steam temperature rating is 400 , and between the \pm 20 fluctuations is normal state. If the steam temperature is slightly higher than 420 , then the parameter judged anomaly immediately is not science, so the normal and abnormal boundaries are fuzzy, therefore the introduction of the concept of fuzzy membership degree for judgment of fault symptom is necessary. The accuracy of fault diagnosis system depends on the accurate judgment for the degree of fault symptoms, and the key to ensure the accuracy is to select the membership function properly, so choosing the appropriate membership function is crucial for the whole system. In industrial fault diagnosis, it usually determine the membership function of fault symptoms into mathematical models and human judgment methods[1]. Regardless of the method to determine the membership function of fault 
symptom, it need to pass the test of practice, analysis of the results of the feedback, and adjust the membership function constantly, then make it closer to the actual situation, which in order to obtain a more accurate parameter membership.

In the boiler troubleshooting analysis, the fault symptoms as the input of neural network are not given by the real-time monitoring parameters directly, they must take the monitoring parameters associated with the failure of high-level logic concept to quantify into a value between 0 and 1 , ie fault symptoms membership, and it used to characterize the degree of fault symptoms. Forms of different fault symptoms are not identical, and the fault symptoms through the actual analysis can be divided into three types: (1) phenotype: which processed directly of the current parameters, and independent of the parameters before and after change, such as the drum pressure is high, the steam temperature is low. (2) process type: which can't directly to process of the current parameters, and the parameters related to the changing time, such as the drum water level rise, the furnace negative pressure drop. (3) relevant type: which associated with several parameters, such as the water flow is less than the steam flow, the water flow is anomaly greater than the steam flow. The fault diagnosis knowledge is usually vague, which described by the fuzzy language is more close to people's way of thinking, and convenient for qualitative analysis. According to the actual situation combined with the experience of experts in boiler, the appropriate fault symptoms membership function is selected. Below to the calculation of single parameter fuzzy membership degree is low. for example, such as the steam pressure is low, exhaust gas temperature is low. The fault membership function formula is as follow:

$$
\mu_{A}(x)= \begin{cases}0 & x \geq a \\ \sqrt{\frac{a-x}{a-b}} & b \leq x<a \\ 1 & x<b\end{cases}
$$

Eq. 1, a is the normal of measured parameters; $b$ is the lower limit of measured parameters;

For example, according to the technical standard of the boiler, the normal value of exhaust gas temperature is 130 , and the lower limit value is 100 . When the exhaust gas temperature of a boiler is 110 , according to the formula (1), the fault membership degree of "the exhaust gas temperature is low" is 0.816 .

\section{BP Neural Network Algorithm and Its Improved Algorithm}

Boiler failure is related to many factors, and there are perplexing relationship among all the factors, so it has certain difficulties to establish a specific mathematical model. In this paper, the boiler is designed as a "black box" model. Real-time data of field collection are processed into a variety of fault symptoms membership through fuzzy logic, when The various fault symptoms as input parameters, the output is the probability of each possible failure of the boiler, namely failure confidence. Fault diagnosis of the boiler is required to use neural network, and BP neural network has a good application in the diagnostic analysis, so it's used for data analysis and processing in this paper.

BP neural network consists of input layer, hidden layer and output layer, and the number of hidden layer is determined according to the specific problems. BP neural network's essence is the use of error back propagation algorithm for multilayer sensor training [2,3]. BP algorithm for weight adjustment method is divided into single sample training and batch training (cycle training). When the number of samples is large, batch training has faster convergence speed. Considering the number of the samples is large, batch training mode is chose for data processing in this system, which can improve the training speed effectively.

The algorithm of BP neural network has strong nonlinear mapping ability, generalization ability, associative self-learning ability and fault-tolerance ability, and the BP neural network has been 
widely used, because it can be arbitrary precision approximation of nonlinear function, but in the application is still exposed many shortcomings. For example, prone to local minimum, so it can't guarantee to find the global optimal solution; Too much training times, resulting in inefficient of learning, and the network convergence speed is slow; the lack of theoretical guidance of the selection for hidden node number; When training the network, it has the trend of forgetting the old samples in learning the new samples[4]. Aiming at these problems, domestic and foreign experts and scholars have put forward a lot of improved BP neural network algorithm, and the methods which often be used are increase the momentum term, the adaptive learning rate, the introduction of steep steps factor and other.

The boiler system is complex, and the samples have highly nonlinear, so the error of gradient is very easy to fall into local minimum in the network training when using the standard BP neural network for the boiler fault diagnosis, which resulting in slowing the convergence speed. In order to improve the network training speed and make the diagnosis fault of the boiler be more consistent with the actual operating conditions, a BP neural network by using the combination of momentum and adaptive learning rate gradient descent method is applied to train the network in this paper, which can improve the diagnosis accuracy.

\section{Create A Fault Diagnosis Model Based on Fuzzy Neural Network}

Fuzzy logic technology has higher processing speed but lower computing power, and the neural network has higher computing power but slower convergence rate, so a combination of both produce a fuzzy neural network. Its remarkable characteristic is that it fully absorbs the advantages of fuzzy logic and the neural network, and make up for their deficiencies[5]. According to the connection between their forms and functions, they can be divided into loose type, parallel type, series type, learning type and the structure equivalent type. Considering the characteristics of fault diagnosis analysis and processing of monitoring data in this system, series type is used in this paper. The application of fuzzy logic is to fuzzy pretreatment on the input parameters of the neural network, and then the parameters which processed are input to the neural network and to be trained, this equivalent of prior to classification of the monitoring data before the network training.

Fault diagnosis model of industrial boiler based on the fuzzy neural network algorithm is divided into training module and diagnosis module. The mainly work of training module is as follow: firstly to obtain fault knowledge, the boiler fault phenomena(fault symptoms sets) and their corresponding fault instances (faults sets) as the study samples of neural network which provided by the experts in related fields and the workers have rich practical experience are assigned to the input nodes and output nodes of the network. Secondly the fault samples are trained through the BP neural network learning algorithm, and the adaptive learning rate gradient descent method is applied to adjust the weights of the network until the error of the actual output and the expected output reaching the required precision, so the fault diagnosis knowledge of the boiler is stored in the BP neural network by a distributed manner. The function of diagnostic module is to change the real-time operation data into the fault symptoms menbership through the fuzzy logic technology, then the fault symptom membership extracted as the input parameters of BP neural network, the output parameter is the degree of confidence of boiler fault. Flow chart of fault diagnosis algorithm based on fuzzy neural network as shown in figure 1 . 


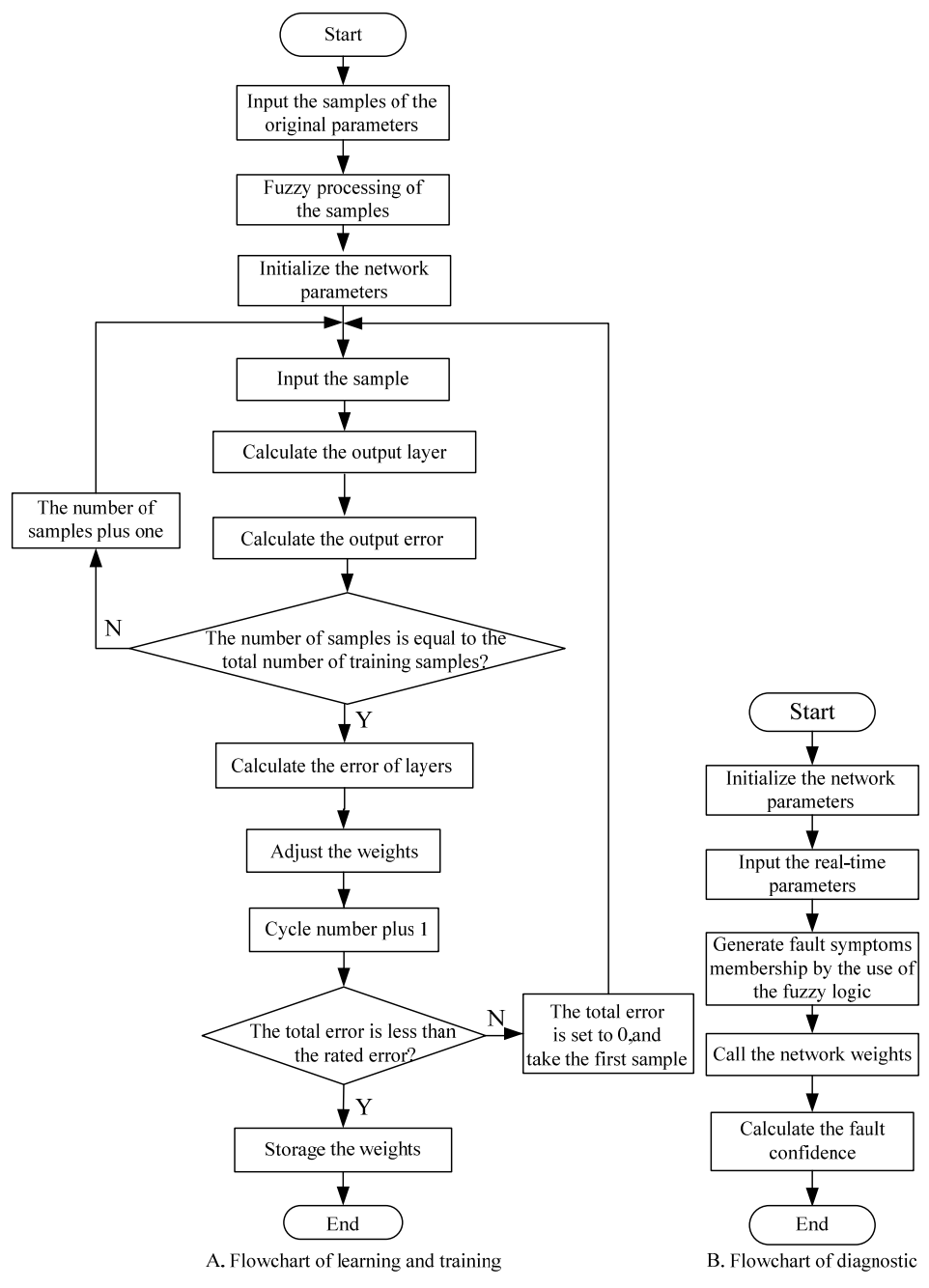

Fig1.Flow chart of fault diagnosis

The relationship between boiler fault and the real-time data is complex, the changes of a plurality of operating parameters can be caused by a fault, in the same one operating parameter changes can cause multiple faults occurring simultaneously. 12 typical kinds of boiler faults are diagnosed through studying on 24 operation parameters which directly related to boiler fault and their dynamic changes in this paper. If they are designed as a single neural network, the network structure and the training samples will be large, which may make difficult for the network training. At the same time the network is sensitive to the sample error, and it's easy to cause the low classification accuracy, which will make inaccurate results for the fault diagnosis. According to the operation characteristics and fault characteristics of the boiler, Each fault is related with certain fault symptoms (real-time monitoring parameters) only, so the unrelated connections between faults and fault symptoms can be removed to reduce the size of the network. Each fault can be mapped with an independent sub network. According to the real-time running parameters collected at the scene, There are 39 fault symptoms put forward to diagnosis and forecast 12 typical kinds of boiler faults, therefore boiler fault diagnosis system which with 12 independent parallel neural networks is composed in this paper. Each input of the sub-network is the fault symptoms of the fault, and the output is the level of confidence for the fault. The use of the parallel network structure can reduce input and output nodes of the network, accelerate the speed of fault diagnosis, and can achieve the simultaneous diagnosis of multiple faults. At the same time, each sub-network is trained independently and without mutual influence. What's more, a sub-network problems will not affect the other sub-network, and easy to modify and supplement of the network. 


\section{Analysis of The Fault Diagnosis Example}

12 typical fault types of the boiler are studied in this paper, which namely full water, water shortage, furnace fire extinguishing, impact boiler's water, the damage of high-temperature superheater, etc. The following is an example of the damage of high temperature superheater, and the application of fuzzy neural network model is for the diagnosis and analysis of this fault.

\section{The Establishment of The Sample}

In determining the inputs of the neural network, the inputs should as far as possible be selected the parameter variables which have great influence and directly linked with the output, and the correlations between the input variables is small or unrelated [6]. Where possible, the parameter variables which have indirect correlation or correlation is negligible should't be chose in order to simplify the network structure and reduce the complexity of the network. The fault symptoms which directly related with the damage of high temperature superheater are low exhaust gas temperature, high fan current, high main steam temperature, low main steam pressure, the furnace negative pressure is reduced into a positive pressure, small main steam flow, and large water flow, so the seven fault symptom memberships above are chose as the neural network input variables. The network is applied to learn and train the damage of high temperature superheater, Therefore, choosing the high temperature superheater damage confidence as the network output.

The generalization ability of the network is affected by the number of samples. In order to get a good generalization ability, the number of samples should be greater than the number of adjustable weights several times generally, so the training result will be more reliable. At the same time, the samples should be representative and the similar samples have diversity and uniformity. The number of high temperature superheater damage fault samples is determined 20 in this paper. Because it's need to use different samples beyond the training set to test the performance of the neural network, Therefore the fault samples are divided into two groups, the former 15 groups as the training set are used for training the network, the 5 groups followed as the testing set are used to test the training results. The table of high temperature superheater fault samples is shown in table 1.

Tab. 1 The table of high temperature superheater fault samples

\begin{tabular}{cccccccccc}
\hline & NO. & $\mathrm{X}_{1}$ & $\mathrm{X}_{2}$ & $\mathrm{X}_{3}$ & $\mathrm{X}_{4}$ & $\mathrm{X}_{5}$ & $\mathrm{X}_{6}$ & $\mathrm{X}_{7}$ & $\mathrm{Y}$ \\
\hline & 1 & 0.525 & 0.280 & 0.835 & 0.800 & 0.585 & 0.500 & 0.313 & 0.05 \\
& 2 & 0.305 & 0.282 & 0.535 & 0.600 & 0.535 & 0.400 & 0.375 & 0.08 \\
& 3 & 0.600 & 0.290 & 0.500 & 0.785 & 0.007 & 0.405 & 0.375 & 0.10 \\
& 4 & 0.885 & 0.372 & 0.655 & 0.800 & 0.785 & 0.465 & 0.265 & 0.25 \\
& 5 & 0.605 & 0.225 & 0.255 & 0.500 & 0.605 & 0.305 & 0.185 & 0.32 \\
Train & 6 & 0.800 & 0.265 & 0.250 & 0.550 & 0.665 & 0.366 & 0.285 & 0.45 \\
set & 7 & 0.925 & 0.205 & 0.207 & 0.585 & 0.605 & 0.305 & 0.288 & 0.63 \\
& 8 & 0.845 & 0.250 & 0.165 & 0.565 & 0.800 & 0.363 & 0.310 & 0.65 \\
& 10 & 1.000 & 0.105 & 0.007 & 0.480 & 0.833 & 0.280 & 0.288 & 0.98 \\
& 11 & 0.880 & 0.240 & 0.155 & 0.580 & 0.007 & 0.300 & 0.315 & 0.62 \\
& 12 & 0.960 & 0.205 & 0.105 & 0.520 & 0.700 & 0.265 & 0.250 & 0.70 \\
& 13 & 0.700 & 0.250 & 0.350 & 0.620 & 0.705 & 0.465 & 0.350 & 0.30 \\
& 14 & 0.550 & 0.335 & 0.005 & 0.800 & 0.007 & 0.485 & 0.300 & 0.10 \\
Test & 15 & 0.560 & 0.315 & 0.800 & 0.825 & 0.560 & 0.500 & 0.315 & 0.05 \\
\hline \multirow{6}{*}{ set } & 16 & 0.520 & 0.275 & 0.755 & 0.835 & 0.557 & 0.520 & 0.363 & 0.06 \\
& 17 & 0.500 & 0.285 & 0.835 & 0.785 & 0.600 & 0.485 & 0.340 & 0.05 \\
& 18 & 0.882 & 0.350 & 0.665 & 0.725 & 0.705 & 0.465 & 0.255 & 0.25 \\
& 19 & 0.762 & 0.282 & 0.435 & 0.655 & 0.715 & 0.485 & 0.310 & 0.26 \\
& 20 & 0.805 & 0.265 & 0.165 & 0.575 & 0.750 & 0.400 & 0.350 & 0.60 \\
\hline
\end{tabular}




\section{Design of The Network Structure}

Theoretical analysis shows that, as long as the BP neural network with 1 hidden layer can realize all of the nonlinear mappings. If increasing the number of layers will make the network more complex and increase the training time of the network weights. Analysis of the design requirements of this network, using the BP neural network of a single hidden layer can meet the requirements.

The number of hidden nodes depends on the number of samples, the size of sample noise and the complexity of sample internal rules. Trial-and-error method is often used to determine the optimal number of hidden nodes. According to the following formulas to determine the initial value:

\section{Error! Reference source not found.}

\section{(2)}

\section{Error! Reference source not found.}

\section{Error! Reference source not found.}

In Eq. $2 \sim$ Eq.4: $\mathrm{j}$ as the number of hidden nodes, $\mathrm{n}$ as the input layer nodes, $\mathrm{m}$ as the output layer nodes, $\mathrm{a}$ is a constant of $1 \sim 10$. According to the trial-and-error method, the corresponding relationship between the hidden nodes and the mean-square error of network is shown in table 2 .

Tab.2 The corresponding relation between the number of hidden nodes and the mean square error

\begin{tabular}{ccccccc}
\hline $\begin{array}{c}\text { Hidden } \\
\text { nodes }\end{array}$ & 2 & 3 & 4 & 5 & 6 & 7 \\
\hline $\begin{array}{c}\text { mean-square } \\
\text { error }\end{array}$ & 0.00051 & 0.00045 & 0.00038 & 0.00035 & 0.00030 & 0.00042 \\
\hline
\end{tabular}

From the table we can get the best hidden nodes is 6 .

In the field of fault diagnosis, the sigmoid function and the hyperbolic tangent function are usually selected as the transfer function. The sigmoid function is applied to the confidence region in the case of $[0,1]$, and the hyperbolic tangent function is applied to the confidence region in the case of $[-1,1]$. Output of this network is the confidence of high temperature superheater damage, which hoped its output in the range of $[0,1]$, so the sigmoid function is chose as the transfer function of output layer, and the transfer function of the output layer generally choose a linear function.

\section{Training and Testing of The Network}

After the establishment of samples and the design of the network structure are completed, the BP neural network is learned and trained by the use of training samples. After training, the fault diagnosis knowledge of the high temperature superheater is distributed in the BP neural network internal weights, which is equivalent to an experienced expert's mind, namely fault knowledge of the network. The inputs of testing set start from the initial state and reasoning forward by the use of the network weights obtained from the training set, then the fault data is predicted from the network. The prediction data is compared with the actual fault data, if they are very close and the error is small, it can be proved that the fault experience and knowledge can be well expressed by the network weights and the weights are right. If the error is large, it can be proved that the fault experience and knowledge can't be well expressed by the network weights and the weights are wrong, and the network must be retrained again. Start training, the initial learning rate is selected as 0.10 , the initial weights are selected the random number between $(-1,1)$. After learning and training, the correct weights of the network are as follows: 


\section{Error! Reference source not found. \\ Error! Reference source not found. \\ Error! Reference source not found.}

$b_{m}=-0.3992$

$\omega_{n j}$ represents the connection weights from the input layer to the hidden layer, $n=1,2, \ldots, 7 ; j=1,2, \ldots, 6$. $b_{j}$ represents the thresholds of the hidden layer. $\omega_{j m}$ represents the connection weights from the hidden layer to the output layer, $m=1 . b_{m}$ represents the thresholds of the output layer. After the network structure and the correct weights are stored, and the training set and test set are learned and trained, the corresponding relationship between the actual outputs and the predictive outputs is shown in Figure 2.

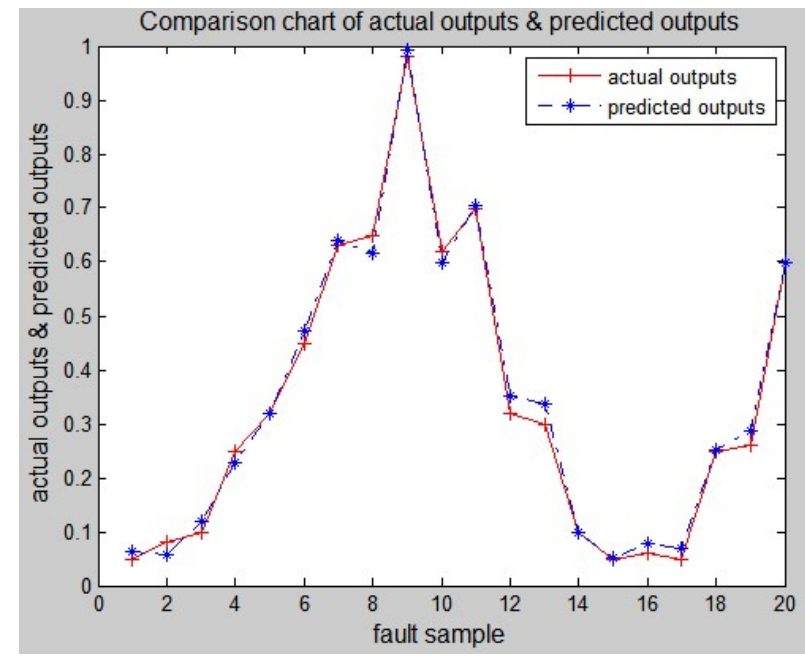

Fig2.the actual outputs and the predictive outputs of high temperature superheater damage

\section{Analysis of Training Results}

Through analysis of Figure 2, the predictive outputs and the actual outputs are match closely. Figure 3 shows the error among the fluctuated between -0.1 to 0.1 , which proves that the network has the right to learn the fault diagnosis knowledge of the boiler, and the results are consistent with the actual situation. The network can diagnose the fault of the boiler and forecast occurrence degree of the boiler fault effectively and accurately.

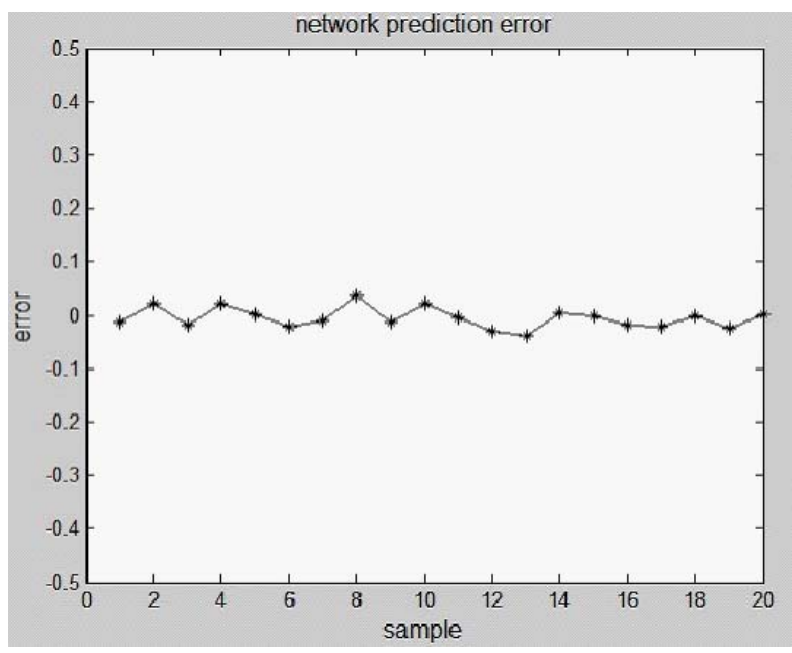

Fig3.Network prediction error of high temperature superheater damage 


\section{Conclusion}

In this paper, a new fault diagnosis model based on the fuzzy neural network is built by the combination of the fuzzy logic technology and the improved BP neural network, and take the damage fault of the high temperature superheater as an example to analysis, which is validation of the fault diagnosis model can simulate human's logical thinking ability, make timely and accurate diagnosis of the boiler fault. With the advantages of clear level, easy to understand, efficiency reasoning, it can greatly reduce the working intensity, and provide an effective guidance for operation .

\section{Reference}

[1] MR.Delgado, F.V.Zuben, F.Gomide, Coevolutionary genetic fuzzy systems:a hierarchical collaborative approach, Fuzzy sets and systems, Vol.141, No.1, pp. 89-106, 2004.

[2] Han Liqun, Artificial neural network tutorial, Beijing University of Posts and Telecommunications press, Beijing, pp. 29-36, 2006.

[3] Niu Peifeng, Zhang Ze, Wang Huaibao, Fault diagnosis research for power plant boiler based on fuzzy clustering and neural networks, Microcomputer information, Vol.26, No.3, pp. 40-42, 2010.

[4] Gao Jun, Artificial neural network theory and simulation, Mechanical industry press, Beijing, pp.44-54, 2005.

[5] Zhang Naiyao, Yan Pingfan, Neural network and fuzzy control, Tsinghua university press, Beijing, pp. 166-173, 2000.

[6] He Bin, Zhao Yongsheng, Zou Jiangfeng, et al, The diagnosis research of boiler fault based on network, Central China electric power, pp.1-5, 2006. 